\title{
The "Dynamic Gravitation of Photons: A Hitherto Unknown Physical Quantity". New Aspects on the Physics of Photons
}

\author{
Guido Zbiral \\ Private Retired Scientist, Klosterneuburg, Austria \\ Email: guido@zbiral.at
}

Received 3 January 2014; revised 2 February 2014; accepted 1 March 2014

Copyright (C) 2014 by author and Scientific Research Publishing Inc.

This work is licensed under the Creative Commons Attribution International License (CC BY). http://creativecommons.org/licenses/by/4.0/

\section{(c) (i) Open Access}

\begin{abstract}
In order to explore the nature of photons, no doubts can be allowed to exist concerning the "physics of photons". While static gravitation plays no role in the physics of photons, this paper will show that the previously unknown nonbaryonic dynamic gravitation of photons determines not only the external physical behaviour of photons but also, in particular, the hitherto unknown physical events occurring within the photons themselves. For this reason, the paper places particular emphasis on dynamic gravitation as a new hitherto unknown physical quantity. Moreover the new type of gravitation postulated here also provides a plausible explanation of the mysterious nonbaryonic dark matter. As no generally accepted scientific explanation of the creation and essence of dark matter exists to date, it is to be anticipated that the nonbaryonic dynamic gravitation of photons is of general interest to physicists as well as cosmologists and may serve to initiate a general debate among them. Furthermore, this paper will also show that there exists a close mutual relationship between electrodynamics <> dynamic gravitation $<>$ static gravitation <> electrostatics <> electrodynamics (refer to paragraph 4). Due to the fact that the insights into the relationship between photons and their dynamic gravitation have not been described by any other author to date, there exists only a few references that I can cite in support of my paper.
\end{abstract}

\section{Keywords}

Photons; Speed of Light; Baryonic (Static) Gravitation; Nonbaryonic Dynamic Gravitation; Nonbaryonic Static Gravitation (Dark Matter); Gravitational Quanta

\section{Introduction}

Note: for the purpose of a better understanding of the following text on the nonbaryonic dynamic gravitational 
nature of photons, it is necessary for the introduction to this paper to mention the "Effects of static gravitation on electromagnetism", which to a large extent corresponds to the content of an earlier paper of mine [1]: "Does Gravitation have an Influence on Electromagnetism?” Journal of Modern Physics, Vol. 3, No. 9 A, (2012) http://www.SciRP.org/journal/jmp

Static gravitation is known as both baryonic gravitation and nonbaryonic gravitation, the latter represents the mysterious "dark matter"; a plausible explanation of the nature of dark matter is described in [2].

All kinds of energy, which are identical with matter $\left(\mathrm{E} \equiv m \cdot c^{2}\right)$, are subject to gravitation; this also applies to electromagnetic energy. Gravitation as an integral (i.e. inseparable) component of each and every form of energy can neither be created nor destroyed. Therefore there must also exist in analogy to the "Law of Conservation of Energy" a "Law of Conservation of Gravitation", described in [1] (paragraph 6). Both opposite acting physical quantities were generated at the same moment in the Big Bang and have the same unknown origin.

Therefore there is no doubt that a fundamental connection exists between electromagnetism and gravitation from the very beginning of the cosmos' existence. However, whether or not this connection needs to be taken into account in the physical theories, such as the Theory of Special Relativity, will be investigated in this paper and offered up for a general debate among the community of physicists. An unambiguous clarification of this alternative question is therefore of key importance, since the physical events within the photons must be known in order to understand the real structure and the nature of photons respectively of the speed of light.

It is possible to derive an initial hint of aphysical relationship between the speed of light $\mathrm{c}$ and the gravitational constant $\mathrm{G}$ from $\mathrm{G}$ itself: Looking at the gravitational constant

$$
G=6.67 \times 10^{-11}\left[\mathrm{~m}^{3} \cdot \mathrm{kg}^{-1} \cdot \mathrm{s}^{-2}\right]
$$

one can see that the physical dimension of $c^{2}\left[\mathrm{~m}^{2} \cdot \mathrm{s}^{-2}\right]$ is contained in this dimension of G!

Thus the gravitational constant $\mathrm{G}$ itself certainly has something in common with the speed of light. It is possible to convert the gravitational constant $\mathrm{G}$ by extrapolating $c^{2}$ :

$$
G=c^{2} \cdot 7.42 \times 10^{-28}\left[\mathrm{~m} \cdot \mathrm{kg}^{-1}\right]=c^{2} \cdot \text { const. or } G / c^{2}=7.42 \times 10^{-28}\left[\mathrm{~m} \times \mathrm{kg}^{-1}\right]=\text { const }
$$

Moreover, the relationship

$$
G / c^{2}=l_{\text {Planck }} / m_{\text {Planck }}=\text { const } \cdot[\mathrm{m} / \mathrm{kg}][3]
$$

also applies, which signifies that $G / c^{2}=$ const. is a "universal united constant of nature" that had applied as far back as the Planckera, during which the state of super gravitation $\left(\boldsymbol{G}_{\text {super }}\right)$ predominated. Therefore for that quantum era, the above equation then becomes the form

$$
G_{\text {super }} / c_{\text {super }}^{2}=\text { const }
$$

The physical facts of the constancy of speed of light independent of the energy of the photons as well as their wave propagation are described in the Maxwell equations, but they cannot provide any statement concerning the reason for the constancy of the speed of light. There is also no directly recognizable connection to gravitation, as the gravitational constant $\mathrm{G}$ does not explicitly occur in Maxwell's equations. According to this, it would seem that electromagnetism (and hence photons) do not have anything to do with gravitation, as confirmed by today's accepted doctrine in physics. However, it is unexpectedly possible to establish a relationship between Maxwell's theory and gravitation:

As according to Maxwell's so-called Fifth Equation, the following physical relationship between the electrical field constant $\varepsilon_{0}$, the magnetic field constant $\mu_{0}$ and the speed of light c exists:

$$
\varepsilon_{0} \cdot \mu_{0}=1 / c^{2}
$$

then $G / c^{2}=$ const, as well as $G \cdot \varepsilon_{0} \cdot \mu_{0}=$ const. $\left[\mathrm{m} \cdot \mathrm{kg}^{-1}\right]$ respectively $G=l_{\text {Planck }} / \varepsilon_{0} \cdot \mu_{0} \cdot m_{\text {Planck }}=$ const . can be written. (Note: I have developed this new formula of $G$ in accordance with [3]).

This means that within Maxwell's Theory electromagnetism is linked via its field constants $\varepsilon_{0}, \mu_{0}$ with the gravitational constant G. Are we to understand this merely as a random coincidence lacking any physical significance, or does this relationship in fact represent an intrinsic connection between these two forces of nature? 
With regard to the relationships stated above, the expression $G / c^{2}=$ const . may possess a far greater physical significance than hitherto assumed.

(Static) Gravitation is known to be the weakest of the four elementary forces in the universe, whereby the term "static" is normally not used in physics literature with regard to gravitation, since only static gravitation is known today. Therefore the (static) gravitational force within an atomic structure between a proton and an electron is weaker than the electromagnetic force acting between them by a factor of $\mathbf{1 0}^{-\mathbf{3 9}}$. For this reason, the gravitational force of the baryonic components of an atom among themselves within an atom can be completely ignored compared to the electromagnetic force between the proton and electron.

This fact forms the basis for the assumption prevalent among physicists that the gravitational force of a photon, compared to its far stronger electromagnetic force, can also be completely ignored, whereby static gravitation is always assumed in this regard, since no dynamic gravitation is so far known. The current school of thoughts in physics is therefore based on the assumption that (static) gravitation is also not a factor to be considered with photons or the speed of light and may be completely ignored.

\section{The Hitherto Unknown "Dynamic Gravitation of Photons"}

For some decades now, physicists have been engaged on research around the globe in fields such as the unification of gravitation and electromagnetism, an explanation for dark matter and dark energy, and the development of a theory of quantum gravitation, etc., but so far to little avail. All this gives the impression that at least one of the assumptions, which underlie either the current doctrine in physics or today's physical and/or cosmological model concepts, must be wrong or incomplete and is preventing a solution of these problems from being found; otherwise one or the other breakthroughs in these fields of physics would already have taken place.

Following the original creation of the cosmos consisting only of radiation energy (electromagnetic primordial photon radiation and the equal strong super gravitational radiation), no "static gravitation" could have existed in the extreme dynamic "Big Bang” phase and shortly afterwards. During the Planck Era the gravitation of the primordial photons was exactly as dynamic in nature as the primordial photons themselves and can therefore only have been created by means of gravitational quanta! The photons' dynamic gravitation remains conserved for the total lifetime of the photons, which are the oldest elementary particles in existence. Their gravitation cannot be inherently static in nature, since it travels with the same speed of light and possesses the same frequency as its associated photon; in other words: "Static gravitation does not exist for photons, since photons are always dynamic"! The physical processes occurring in the earliest phase of the cosmos during the Planck era (in a state of super gravitation), are even today still taking place in the photons (in qualitative terms), virtually representing a "remnant left over from that quantum era".

From the arguments made above it follows, that each photon must have emerged as a quantum of electromagnetic energy from the Big Bang with its quite specific (photon energy equivalent) quantum of gravitation, with which it appears together and which causes the dynamic gravitation of the photon as well as its relativistic (i.e. nonbaryonic) mass. This specific quantum of gravitation does in fact resonate with the same frequency as the photon itself, because it is "directed" by the latter; therefore it appears as a high frequency gravitational radiation locally bound to its photon. It remains closely associated with the photon until the photon is expended or transformed by performing work. As on this occasion the gravitation of the expended photon cannot disappear or be destroyed (Law of Conservation of Gravitation), in fact the quantum of dynamic gravitation is converted into the transformation product as weak static gravitation [2]. This opens up a completely new insight into the physics of photons!

The dynamic gravitation of photons differs generally from the static gravitation of baryonic matter, since the dynamic gravitation of photons is nonbaryonic in nature; otherwise it could not travel with the photon at the constant speed of light.

Therefore, in my opinion, the assumption underlying the current doctrine of physics of a negligible static gravitation of photons is not only erroneous but also entirely devoid of meaning, since it does not exist! In contrast to the prevalent school of thoughts in physics, I therefore hold the view that dynamic gravitation in the form of local gravitational radiation must definitely be taken into account with regard to photons.

A photon without local dynamic gravitation does not exist. Thus each photon consists of two components closely linked to each other, one expansive electromagnetic radiation component (quantum) and one 
gravitational radiation component (quantum) acting precisely in an opposite manner. These two physical quantities are ideally united in the photon. Therefore there must exist exactly the same number of nonbaryonic gravitational quanta as there are of photons themselves.

\section{Effects of the Nonbaryonic Dynamic Gravitation of Photons}

The energy of photons is known to increase proportionally with its frequency $v$ in accordance with Planck's equation

$$
E=h \cdot v
$$

where $h=6.626 \times 10^{-34}$ Js is Planck's constant or "effect-quantum". As electromagnetic radiation encompasses an extremely broad frequency spectrum, the energy of the photons also occupies this very wide range. Notwithstanding this, all photons always travel in a vacuum with the same constant speed of light, irrespective of the energy quantum that they transport with them. Each photon requires its entire positive electromagnetic energy for the purpose of maintaining the constant speed of light against the negative stabilising braking force of their dynamic gravitation. The photon travelling at the speed of light does not have any "free energy" available for any additional "external" effect; their sole external effect is the constancy of speed of light. Therefore the constant speed of light must be associated with the wide ranging variable relativistic mass of photons in such a manner that the photon rich in energy (=relativistic mass) must be subject to the stabilising braking force of gravity to a greater extent than the photon low in energy, thus always cancelling out the two opposing forces of nature with the result, that at the speed of light the total energy of each photon is always zero.

Therefore the constant speed of light can only represent a stable state of equilibrium between the expansive force of electromagnetic radiation of the photons and the opposing force of the photons dynamic gravitation.

There is no way for this equilibrium and hence for the constancy of the speed of light to be achieved except by the nonbaryonic dynamic gravitation of the photons.

On its own (without dynamic gravitation) electromagnetism is unable to produce a stable state of equilibrium. This is the evidence that each photon as a quantum of electromagnetic energy is connected with an equivalent quantum of gravitational energy. In the micro world of photons, their dynamic gravitation is unambiguously a force and not a geometry; if this were not so, the unification of dynamic gravitation with the dynamic force of electromagnetism would not be possible.

What happens inside the photons, i.e., what physical process must take place in order for the speed of light to be constant irrespective of the energy of the photons? There must be in fact a very fast regulations process at work, i.e. the speed of light is regulated to the constant value of $\mathrm{c}$ by a hitherto unnoticed "influencing factor", located within each individual photon. As an "influencing factor", however, the only candidate is thus the nonbaryonic dynamic gravitation of the photons themselves, since any change in energy of a photon simultaneously causes an equal change in its relativistic mass as well as in its dynamic gravitation. This change in dynamic gravitation affects a photon to the extent that it is precisely the stabilising effect of gravitation corresponding to the actually existing energy state available out of all the energy states possible. Therefore the speed of light remains constant for all possible energy situations of a photon.

In order for a state of equilibrium to result from the two dynamic forces acting on the photon, the dynamic gravitational field must exhibit a behaviour precisely opposite to that of the dynamic electromagnetic field.

Therefore the physical principles of both radiation fields must be in every respect completely equivalent but opposed to each other. It therefore necessarily follows that the same vectorial Maxwellian Theory must also underlie the nonbaryonic gravitational radiation associated with the photon as is the case with the electromagnetic radiation of the photon. Both physical quantities of each photon would then thereby possess a common theoretical basis!

Following the same logic, gravitational radiation must possess transversal characteristics exactly as electromagnetic radiation does, and it must also exhibit the same frequency as electromagnetic radiation, and this is indeed the case. It is only as a consequence of these conditions that there exists no resulting force on the photon causing it to accelerate or decelerate. Therefore the photon travels in a completely uniform manner at the constant speed of light. The constancy of the speed of light is therefore the result of an extremely finely-tuned interaction between the photons' dynamic gravitation and their dynamic electromagnetism, from which it follows that at the speed of light there exists a close relationship between these two opposite dynamic 
forces of nature:

On one hand, the photons' nonbaryonic dynamic gravitation limits the speed of expansion of electromagnetic energy (by establishing a state of equilibrium between the two forces of nature) and thus determining the value of the speed of light $c$ with $299,792,458 \mathrm{~m} / \mathrm{s}$ in a vacuum, while, on the other hand, maintaining it at this constant value by an internal fast regulation process!

Since the energy of the electromagnetic radiation is proportional to its frequency according to the equation $E=h \cdot v$, it is required that the energy of the high frequency gravitational radiation associated with a photon also be proportional to its frequency, whereby both frequencies are identical. This is necessary to achieve a stable state of equilibrium at the speed of light.

Therefore Planck's equation $E=h \cdot v$ must also apply to the high frequency gravitational radiation, associated with each photon and only selectively (local) acting on it. As previously mentioned above, it then follows that the energy of the gravitational radiation associated with each photon must similarly be quantised.

This fact, combined with the statements regarding dynamic gravitation of photons made above, might possibly open an insight towards a Theory of Quantum Gravitation. Hence it follows, that the quantum gravitation of each photon is exactly as dynamic as the respective electromagnetic quantum itself.

Although static gravitation is the weakest of the four fundamental forces in the universe, it is surprising to discover here, that at the speed of light the dynamic gravitation of a photon is exactly as strong as the dynamic electromagnetism of a photon itself, but acting in an opposite manner, so that both of these fundamental forces exist in a stable state of equilibrium cancelling each other out from an external perspective. As the identical situation also existed with respect to the primordial photons in the state of supergravitation, the dynamic gravitation of photons today thus represents nothing other than the reflection of the dynamic supergravitation during the Planck Era.

\section{Horizontal and Vertical Relationships between Electromagnetism and Gravitation}

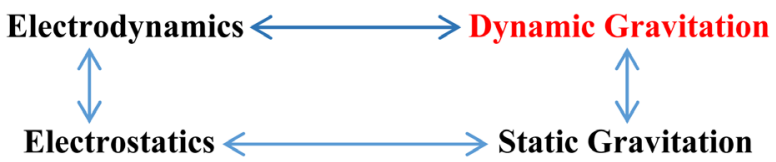

As is evident from the above, there exists a close "horizontal relationship" between the two opposed dynamic forces of nature, since they both exhibit completely identical physical properties. On this basis, it can be concluded that the same horizontal relationship also exists between electrostatics and static gravitation, as reflected in the similar structure of their formulae for static forces.

Note: In addition, in a lecture to the Congress of the German Physical Society in 2012 in Göttingen, K. O. Greulich [4] has also established that electrostatics and (static) gravitation are alike from a physical perspective!

A. I. Arbab [5] of the University of Khartoum confirms that an analogy exists between electromagnetism and gravity; the two opposite quantities represent unified phenomena.

Furthermore, there also exists a close "vertical relationship" between each of the two opposed dynamic quantities and their corresponding static quantities, since the latter are "descended" in each case from their dynamic quantities. Dynamic gravitation, however, from which static gravitation is derived, has surprisingly remained unknown to date. When the pervasive symmetry in nature and, in particular, in the laws of nature is taken into account, then it is reasonable to conclude, that in analogy to the static quantities of "electrostatics and static gravitation” two dynamic quantities also ought to exist, i.e. dynamic gravitation in addition to electrodynamics. In this regard, a “dynamic counterpart to static gravitation” is evidently missing.

As previously stated above, each photon consists of two dynamic components or quanta, acting precisely in an opposite manner at the speed of light. Both components together constitute each and every photon as an inseparable unity of effect. Therefore the dynamic gravitation cannot be "discovered" as an isolated physical entity, since it is inherently strictly bound to the electromagnetic component of each photon. For this reason, it is hidden from the external world, it only being possible to infer its existence from its "external effects". As previously mentioned, the external effects of dynamic gravitation are the value and the constancy of speed of light. Another external effect is the so-called "dark matter" [2].

The nonbaryonic dynamic gravitation of photons and its conversion into nonbaryonic static gravitation as 
a result of an energy transformation of photons (by performing external work, particularly during the Big Bang and the Planck Era) provides a plausible explanation for the mysterious "nonbaryonic dark matter". If nonbaryonic dark matter really can be explained by means of the nonbaryonic dynamic gravitation of photons, then I would regard it as a confirmation for the correctness of the thoughts expressed here on the "New Aspects on the Physics of Photons".

\section{Unification of Gravitation and Electromagnetism}

A photon's electromagnetic radiation forms a physical dualism with the gravitational radiation associated with it (complete equality of both counteracting components linked to each other).

The unification of the nonbaryonic dynamic gravitation and the dynamic electromagnetism, sought in vain for decades, is only possible if the two opposing physical forces are based on a common theory, in our case Maxwell's Theory; both physical quantities possess the same structure and are perfectly united and unified in each photon on the common basis of their energy $E=h \cdot v$ !

The unification of gravitation with electromagnetism has already clearly been expressed at the beginning of this paper in the equation $G / c^{2}=G \cdot \varepsilon_{0} \cdot \mu_{0}=$ const. [m/kg] .

\section{Conclusions}

The gravitation of a photon is as dynamic in nature as the photon itself. The assumption underlying the current doctrine of physics of a negligible static gravitation of photons is erroneous.

The hitherto unknown nonbaryonic dynamic gravitation of photons determines their physical properties and must therefore be taken into account with all relevant considerations.

Dynamic gravitation is produced by gravitational quanta and appears in the form of gravitational radiation locally bound to each photon. Therefore there must exist exactly the same number of gravitational quanta as there are of photons.

With respect to its energy, both electrodynamics and dynamic gravitational radiation possess Maxwell's Theory as a common theory. Therefore the energies of both opposing components are of equal strength $(\boldsymbol{E}=\boldsymbol{h} \cdot \boldsymbol{v})$.

At the constant speed of light, a stable state of equilibrium exists within each photon between the expansive force of electrodynamics and the equal but opposing (braking) force of dynamic gravitation. Therefore at the constant speed of light, the resulting total energy of every photon is always zero! This is an absolute necessity for the constancy of speed of light.

In the micro world of photons, their dynamic gravitation is unambiguously a force and not a geometry.

\section{Acknowledgements}

My warmest thanks go to my translator C. A. Szwaja (M.A. Physics, Oxon), both for translating my manuscript from German into English and his numerous valuable suggestions on the text itself.

\section{References}

[1] Zbiral, G. (2012) Journal of Modern Physics, 3, 1223-1230. http://dx.doi.org/10.4236/jmp.2012.329158 http://www.SciRP.org/journal/jmp

[2] Zbiral, G. (2013) International Journal of Theoretical and Applied Physics (IJTAP), 3, 1-14.

[3] Greulich, K.O. (2011) Understanding the Masses of Elementary Particles-A Step towards Understanding the Massless Photons. Proceedings of SPIE, 08121, 1-9. http://dx.doi.org/10.1117/12.892277

[4] Greulich, K.O. (2006) Expression of the Dimensionless Constants of Nature as a Function of Photon and Electron Properties. Spring Meeting of the Deutsche Physikalische Gesellschaft Munich 2006, 20-24 March.

[5] Arbab, A.I. (2012) Journal of Modern Physics, 3, 1231-1235. http://dx.doi.org/10.4236/jmp.2012.329159 http://www.SciRP.org/journal/jmp 


\section{Appendix}

Note: As the contents of this paper dealing with the nonbaryonic dynamic gravitation of photons represents a completely new area of research without any previous existing works to refer to, it is therefore only possible to list a few references. 